\title{
Pseudo-almost periodic solutions for first-order neutral differential equations
}

\author{
Yuehua Yu' and Shuhua Gong ${ }^{2 *}$ (i)
}

\author{
"Correspondence: \\ shuhuagong@aliyun.com \\ ${ }^{2}$ College of Mathematics, Physics \\ and Information Engineering, \\ Jiaxing University, Jiaxing, P.R. China \\ Full list of author information is \\ available at the end of the article
}

\begin{abstract}
In this paper, we study a class of first-order neutral differential equations with time-varying delays and coefficients. Employing the fixed point method and differential inequality techniques, easily verifiable delay-independent criteria are established to ensure the existence and global exponential stability of pseudo-almost periodic solutions for the addressed equations. These theoretical results are also supported with numerical simulations.
\end{abstract}

MSC: $34 \mathrm{C} 25 ; 34 \mathrm{~K} 13$

Keywords: Pseudo-almost periodic solution; First-order neutral differential equation; Existence; Global exponential stability

\section{Introduction}

In [1], Komanovskii and Nosov proposed the following first-order neutral differential equations:

$$
\left[x(t)-c x\left(t-r_{1}\right)\right]^{\prime}=-Q(t) x(t)+f\left(t, x\left(t-r_{2}\right)\right),
$$

to describe the distributed networks containing lossless transmission lines. Here $f$ is a continuous function on $\mathbb{R}^{2}, Q$ is a continuous function on $\mathbb{R}, x(t)$ represents the state variable, $r_{1}$ and $r_{2}$ designate the transmission delays. The detailed biological explanations of the parameters of (1.1) can be found in $[1,2]$. It is well known that the variation of the environment plays an important role in many biological and ecological dynamical systems. In particular, periodically varying environment and almost periodically varying environment are foundations for the theory of nature selection. Furthermore, (1.1) has been naturally generalized as the following first-order neutral differential equations with time-varying delays and coefficients:

$$
\left[x(t)-P(t) x\left(t-\tau_{1}(t)\right)\right]^{\prime}=-Q(t) x(t)+f\left(t, x\left(t-\tau_{2}(t)\right)\right),
$$

where $Q, P \in C(\mathbb{R},(0,+\infty)), \tau_{1}, \tau_{2} \in C(\mathbb{R},[0,+\infty))$ are bounded, and $f \in C(\mathbb{R} \times \mathbb{R}, \mathbb{R})$.

Recently, the existence and stability of periodic solutions or pseudo-almost periodic solutions of (1.2) and its generalized equations have been extensively studied. For example, criteria ensuring the existence of periodic solutions are established in [3-9] and some

(c) The Author(s) 2018. This article is distributed under the terms of the Creative Commons Attribution 4.0 International License (http://creativecommons.org/licenses/by/4.0/), which permits unrestricted use, distribution, and reproduction in any medium, provided you give appropriate credit to the original author(s) and the source, provide a link to the Creative Commons license, and indicate if changes were made. 
sufficient conditions for the existence of pseudo-almost periodic (mild) solutions are obtained in $[10,11]$. On the other hand, the global exponential stability of pseudo-almost periodic solutions plays a key role in characterizing the dynamical behavior of biological and ecological dynamical systems since the exponential convergence rate can be unveiled [12-20]. However, to the best of our knowledge, no such work has been performed on the dynamic analysis of pseudo-almost periodic solution of first-order neutral differential equations with time-varying delays and coefficients. With this motivation, our goal is to study the existence, uniqueness and global exponential stability of pseudo-almost periodic solutions of (1.2). Here, a new approach will be developed to obtain the global exponential convergence for the pseudo-almost periodic solutions.

Throughout this brief article, we denote

$$
\begin{aligned}
& w^{+}=\sup _{t \in \mathbb{R}}|w(t)|, \quad w^{-}=\inf _{t \in \mathbb{R}}|w(t)|, \\
& r=\min \left\{\tau_{1}^{-}, \tau_{2}^{-}\right\}>0, \quad \tau=\max \left\{\tau_{1}^{+}, \tau_{2}^{+}\right\} .
\end{aligned}
$$

The initial condition associated with (1.2) is of the form

$$
x=\phi(s), \quad s \in[-\tau, 0], \phi \in C([-\tau, 0], \mathbb{R}) .
$$

\section{Preliminary results}

In this section, a few lemmas, notations and assumptions are cited which will be used in Sect. 3.

Assume that $\mathbb{B}(\mathbb{R}, \mathbb{R})$ represents the set of all bounded and continuous functions from $\mathbb{R}$ to $\mathbb{R}$. Then $(\mathbb{B}(\mathbb{R}, \mathbb{R}),\|\cdot\|)$ is a Banach space, where $\|\cdot\|$ denotes the supremum norm $\|w\|:=\sup _{t \in \mathbb{R}}|w(t)|$.

Definition 2.1 (see $[21,22]) u(t) \in \mathbb{B}(\mathbb{R}, \mathbb{R})$ is said to be almost periodic on $\mathbb{R}$ if, for any $\varepsilon>0$, there exists a real number $l=l(\varepsilon)>0$ with the property that, for any interval with length $l(\varepsilon)$, it is possible to find a number $\delta=\delta(\varepsilon)$ in this interval such that $|u(t+\delta)-u(t)|<$ $\varepsilon$ for all $t \in \mathbb{R}$.

Let $A P(\mathbb{R}, \mathbb{R})$ be the set of the almost periodic functions from $\mathbb{R}$ to $\mathbb{R}$, and

$$
P A P_{0}(\mathbb{R}, \mathbb{R})=\left\{g \in \mathbb{B}(\mathbb{R}, \mathbb{R})\left|\lim _{T \rightarrow+\infty} \frac{1}{2 T} \int_{-T}^{T}\right| g(t) \mid d t=0\right\} .
$$

A function $\varphi \in \mathbb{B}(\mathbb{R}, \mathbb{R})$ is called pseudo-almost periodic if it can be expressed as $\varphi=h+g$, where $h \in A P(\mathbb{R}, \mathbb{R})$ and $g \in P A P_{0}(\mathbb{R}, \mathbb{R})$. Furthermore, $(P A P(\mathbb{R}, \mathbb{R}),\|\cdot\|)$ is a Banach space and $A P(\mathbb{R}, \mathbb{R})$ is a proper subspace of $P A P(\mathbb{R}, \mathbb{R})[22]$.

Definition 2.2 (see [22, p. 59]) Suppose that $\Omega \subset \mathbb{R}$ and $K$ is any compact subset of $\Omega$. Let $P A P_{0}(\mathbb{R} \times \Omega, \mathbb{R})$ consist of all bounded and continuous functions $\phi$ such that

$$
\lim _{T \rightarrow+\infty} \frac{1}{2 T} \int_{-T}^{T}|\phi(t, z)| d t=0
$$

uniformly with respect to $z \in K$. Let $P A P(\mathbb{R} \times \Omega, \mathbb{R})$ designate all functions $\eta$ of the form

$$
\eta(t, x)=\phi_{1}(t, x)+\phi_{2}(t, x)
$$


where $\phi_{2} \in P A P_{0}(\mathbb{R} \times \Omega, \mathbb{R})$, and $\phi_{1}$ is an almost periodic function for $t$ uniformly on $\Omega$.

In order to study the pseudo-almost periodic solutions for (1.2), we shall assume that $Q \in A P(\mathbb{R}, \mathbb{R}), P, \tau_{1}, \tau_{2} \in P A P(\mathbb{R}, \mathbb{R}), f \in P A P(\mathbb{R} \times \mathbb{R}, \mathbb{R}), \tau_{1}$ and $P$ are uniformly continuous on $\mathbb{R}$, and

$$
M[Q]=\lim _{T \rightarrow+\infty} \frac{1}{T} \int_{t}^{t+T} Q(s) d s>0 \quad \text { for all } t \in \mathbb{R} .
$$

Lemma 2.1 (see [20, Lemma 2.3]) Set

$$
B=\{\varphi \mid \varphi \in P A P(\mathbb{R}, \mathbb{R}) \text { is uniformly continuous on } \mathbb{R}\} .
$$

Then $B$ is a closed subset of $P A P(\mathbb{R}, \mathbb{R})$.

Lemma 2.2 Every solution $x(t)$ of (1.2) with initial value condition (1.4) exists and is unique on $[0,+\infty)$.

Proof Let us show initially that $x(t)$ exists and is unique on $[0, r]$. In fact, for $t \in[0, r]$, let

$$
y(t)=x(t)-P(t) x\left(t-\tau_{1}(t)\right)
$$

and

$$
b(t)=-Q(t) P(t) \phi\left(t-\tau_{1}(t)\right)+f\left(t, \phi\left(t-\tau_{2}(t)\right)\right) .
$$

Then

$$
y^{\prime}(t)=-Q(t) y(t)-Q(t) P(t) x\left(t-\tau_{1}(t)\right)+f\left(t, x\left(t-\tau_{2}(t)\right)\right)=-Q(t) y(t)+b(t)
$$

and

$$
\begin{aligned}
& y(t)=y(0) e^{-\int_{0}^{t} Q(u) d u}+\int_{0}^{t} e^{-\int_{s}^{t} Q(u) d u} b(s) d s, \\
& x(t)=y(t)+P(t) \phi\left(t-\tau_{1}(t)\right) .
\end{aligned}
$$

Hence, $x(t)$ exists and is unique on $[0, r]$. Consequently, the lemma follows from the induction.

\section{Main results}

In this section, we establish the existence and global exponential stability of pseudo-almost periodic solutions of (1.2) by using the fixed point theorem and Lyapunov functional method.

Theorem 3.1 Assume that the following conditions hold.

$\left(A_{1}\right)$ There exist a positive constant $K^{*}$ and a bounded and continuous function $\tilde{Q}: \mathbb{R} \rightarrow$ $(0,+\infty)$ such that

$$
e^{-\int_{s}^{t} Q(u) d u} \leq K^{*} e^{-\int_{s}^{t} \tilde{Q}(u) d u} \quad \text { for all } t, s \in \mathbb{R} \text { and } t-s \geq 0 .
$$


$\left(A_{2}\right)$ There exist positive constants $L^{f}$ and $L$ such that

$$
\begin{aligned}
& \left|f\left(t, x_{1}\right)-f\left(t, x_{2}\right)\right| \leq L^{f}\left|x_{1}-x_{2}\right| \quad \text { for all } t, x_{1}, x_{2} \in \mathbb{R} \\
& \sup _{t \in \mathbb{R}} K^{*} \frac{|Q(t) P(t)|+L^{f}}{\tilde{Q}(t)} \leq L, \quad L+P^{+}<1
\end{aligned}
$$

and

$$
\sup _{t \in \mathbb{R}}\left\{-\tilde{Q}(t)+K^{*} \frac{1}{1-P^{+}}\left[|P(t) Q(t)|+L^{f}\right]\right\}<0 .
$$

Then Eq. (1.2) has a unique pseudo-almost periodic solution, and the solution $x(t)$ of (1.2) with initial condition (1.4) converges exponentially to the pseudo-almost periodic solution as $t \rightarrow+\infty$.

Proof Set $\varphi \in B$ and $F(t, z)=\varphi(t-z)$. According to Theorem 5.3 in [22, p. 58] and Definition 2.2, the uniform continuity of $\varphi$ entails that $F \in P A P(\mathbb{R} \times \Omega, \mathbb{R})$ and $F$ is continuous in $z \in L$ and uniformly in $t \in \mathbb{R}$ for all compact subset $L$ of $\Omega \subset \mathbb{R}$. This, together with $\tau_{i} \in P A P(\mathbb{R}, \mathbb{R})$ and Theorem 5.11 in [22, p. 60], involves

$$
\varphi\left(t-\tau_{i}(t)\right) \in P A P(\mathbb{R}, \mathbb{R}), \quad i=1,2 .
$$

By Corollary 5.12 in $[22$, p. 61] and the fact that $f \in P A P(\mathbb{R} \times \mathbb{R}, \mathbb{R})$, we can show

$$
f\left(t, \varphi\left(t-\tau_{2}(t)\right) \in \operatorname{PAP}(\mathbb{R}, \mathbb{R})\right.
$$

For any $\varphi \in B$, we consider an auxiliary equation

$$
x^{\prime}(t)=-Q(t) x(t)-Q(t) P(t) \varphi\left(t-\tau_{1}(t)\right)+f\left(t, \varphi\left(t-\tau_{2}(t)\right)\right) .
$$

In view of the fact that $M[Q]>0$, it follows from Theorem 2.3 in [23] that the system (3.4) has exactly one pseudo-almost periodic solution

$$
x^{\varphi}(t)=\int_{-\infty}^{t} e^{-\int_{s}^{t} Q(u) d u}\left[-Q(s) P(s) \varphi\left(s-\tau_{1}(s)\right)+f\left(s, \varphi\left(s-\tau_{2}(s)\right)\right)\right] d s, \quad \forall \varphi \in B,
$$

where

$$
\begin{aligned}
{\left[x^{\varphi}(t)\right]^{\prime}=} & \left\{\int_{-\infty}^{t} e^{-\int_{s}^{t} Q(u) d u}\left[-Q(s) P(s) \varphi\left(s-\tau_{1}(s)\right)+f\left(s, \varphi\left(s-\tau_{2}(s)\right)\right)\right] d s\right\}^{\prime} \\
= & -Q(t)\left\{\int_{-\infty}^{t} e^{-\int_{s}^{t} Q(u) d u}\left[-Q(s) P(s) \varphi\left(s-\tau_{1}(s)\right)+f\left(s, \varphi\left(s-\tau_{2}(s)\right)\right] d s\right\}\right. \\
& -Q(t) P(t) \varphi\left(t-\tau_{1}(t)\right)+f\left(t, \varphi\left(t-\tau_{2}(t)\right)\right) \\
= & -Q(t) x^{\varphi}(t)-Q(t) P(t) \varphi\left(t-\tau_{1}(t)\right)+f\left(t, \varphi\left(t-\tau_{2}(t)\right)\right) .
\end{aligned}
$$

Now, we define a mapping $T: B \longrightarrow A P(\mathbb{R}, \mathbb{R})$ as follows:

$$
(T \varphi)(t)=P(t) \varphi\left(t-\tau_{1}(t)\right)+x^{\varphi}(t), \quad \forall \varphi \in B .
$$


Next, we prove that the mapping $T$ is a contraction mapping on $B$.

For all $t \in \mathbb{R}$, (3.6) entails that $\left[x^{\varphi}(t)\right]^{\prime}$ is bounded on $\mathbb{R}$, and $x^{\varphi}(t)$ is uniformly continuous on $\mathbb{R}$. This, together with the uniform continuities of $\tau_{1}$ and $P$, implies that $P(t) \varphi\left(t-\tau_{1}(t)\right) \in B$, and the mapping $T$ is a self-mapping from $B$ to $B$.

Furthermore, for all $\varphi, \psi \in B$, (3.5), $\left(A_{1}\right)$ and $\left(A_{2}\right)$ yield

$$
\begin{aligned}
\| T \varphi & -T \psi \| \\
\leq & \sup _{t \in \mathbb{R}}\left\{\left|P(t)\left[\varphi\left(t-\tau_{1}(t)\right)-\psi\left(t-\tau_{1}(t)\right)\right]\right|\right. \\
& +\int_{-\infty}^{t} e^{-\int_{s}^{t} \tilde{Q}(u) d u} K^{*} \mid-Q(s) P(s)\left(\varphi\left(s-\tau_{1}(s)\right)-\psi\left(s-\tau_{1}(s)\right)\right) \\
& \left.+\left(f\left(s, \varphi\left(s-\tau_{2}(s)\right)\right)-f\left(s, \psi\left(s-\tau_{2}(s)\right)\right)\right) \mid d s\right\} \\
\leq & \|\varphi-\psi\|\left\{P^{+}+\sup _{t \in \mathbb{R}} \int_{-\infty}^{t} e^{-\int_{s}^{t} \tilde{Q}(u) d u} K^{*}\left[|Q(s) P(s)|+L^{f}\right] d s\right\} \\
\leq & \|\varphi-\psi\|\left[P^{+}+\sup _{t \in \mathbb{R}} \int_{-\infty}^{t} e^{-\int_{s}^{t} \tilde{Q}(u) d u} \tilde{Q}(s) L d s\right] \\
\leq & \left(P^{+}+L\right)\|\varphi-\psi\| .
\end{aligned}
$$

Thus, the mapping $T$ is a contraction on $B$. Using Theorem 0.3 .1 of [24], we see that the mapping $T$ possesses a unique fixed point $x^{*} \in B, T x^{*}=x^{*}$, i.e.,

$$
\begin{aligned}
x^{*}(t)= & P(t) x^{*}\left(t-\tau_{1}(t)\right)+x^{x^{*}}(t) \\
= & P(t) x^{*}\left(t-\tau_{1}(t)\right) \\
& +\int_{-\infty}^{t} e^{-\int_{s}^{t} Q(u) d u}\left[-Q(s) P(s) x^{*}\left(s-\tau_{1}(s)\right)+f\left(s, x^{*}\left(s-\tau_{2}(s)\right)\right)\right] d s,
\end{aligned}
$$

which, together with (3.6) leads to

$$
\left[x^{*}(t)-P(t) x^{*}\left(t-\tau_{1}(t)\right)\right]^{\prime}=-Q(t) x^{*}(t)+f\left(t, x^{*}\left(t-\tau_{2}(t)\right)\right),
$$

and $x^{*}(t)$ is a pseudo almost periodic solution of equation (1.2).

Finally, we prove that $x^{*}(t)$ is globally exponentially stable.

Suppose that $x(t)$ is an arbitrary solution of (1.2) associated with initial value $\phi \in$ $C([-\tau, 0], \mathbb{R})$. We trivially extend $x(t)$ to $\mathbb{R}$ by letting $x(t)=x(-\tau)$ for $t \in(-\infty,-\tau]$. Let

$$
y(t)=x(t)-x^{*}(t), \quad Y(t)=y(t)-P(t) y\left(t-\tau_{1}(t)\right) \quad \text { for all } t \in \mathbb{R} .
$$

Then $y(t)$ and $Y(t)$ are bounded and continuous on $(-\infty, 0]$. From (1.2), we have

$$
\begin{aligned}
Y^{\prime}(t)= & {\left[y(t)-P(t) y\left(t-\tau_{1}(t)\right)\right]^{\prime} } \\
= & -Q(t) Y(t)-Q(t) P(t) y\left(t-\tau_{1}(t)\right) \\
& +\left[f\left(t, x\left(t-\tau_{2}(t)\right)\right)-f\left(t, x^{*}\left(t-\tau_{2}(t)\right)\right)\right] .
\end{aligned}
$$


According to (3.3), it is possible to find a constant $\lambda \in\left(0, \tilde{Q}^{-}\right)$satisfying

$$
\sup _{t \in \mathbb{R}}\left\{\lambda-\tilde{Q}(t)+K^{*}\left[\frac{e^{\lambda \tau_{1}^{+}}}{1-P^{+} e^{\lambda \tau_{1}^{+}}}|P(t) Q(t)|+L^{f} \frac{e^{\lambda \tau_{2}^{+}}}{1-P^{+} e^{\lambda \tau_{1}^{+}}}\right]\right\}<0 .
$$

Define

$$
\|\phi\|_{\xi}=\sup _{t \leq 0}\left|y(t)-y\left(t-\tau_{1}(t)\right)\right|
$$

For any $\varepsilon>0$, we can choose a sufficiently large constant $M$ such that

$$
\begin{aligned}
& |Y(0)|<\left(\|\phi\|_{\xi}+\varepsilon\right)<M\left(\|\phi\|_{\xi}+\varepsilon\right), \\
& |Y(t)|<\left(\|\phi\|_{\xi}+\varepsilon\right) e^{-\lambda t}<M\left(\|\phi\|_{\xi}+\varepsilon\right) e^{-\lambda t} \quad \text { for all } t \in(-\infty, 0],
\end{aligned}
$$

and

$$
M>1+K^{*} .
$$

In the following, we will show

$$
|Y(t)|<M\left(\|\phi\|_{\xi}+\varepsilon\right) e^{-\lambda t} \quad \text { for all } t>0 .
$$

Otherwise, there must exist $\theta>0$ such that

$$
\left\{\begin{array}{l}
|Y(\theta)|=M\left(\|\phi\|_{\xi}+\varepsilon\right) e^{-\lambda \theta} \\
|Y(t)|<M\left(\|\phi\|_{\xi}+\varepsilon\right) e^{-\lambda t} \quad \text { for all } t \in(-\infty, \theta)
\end{array}\right.
$$

Furthermore,

$$
\begin{aligned}
e^{\lambda v}|y(v)| & \leq e^{\lambda v}\left|y(v)-P(v) y\left(v-\tau_{1}(v)\right)\right|+e^{\lambda v}\left|P(v) y\left(v-\tau_{1}(v)\right)\right| \\
& \leq e^{\lambda v}|Y(v)|+P^{+} e^{\lambda \tau_{1}^{+}} e^{\lambda\left(v-\tau_{1}(v)\right)}\left|y\left(v-\tau_{1}(v)\right)\right| \\
& \leq M\left(\|\varphi\|_{\xi}+\varepsilon\right)+P^{+} e^{\lambda \tau_{1}^{+}} \sup _{s \in(-\infty, t]} e^{\lambda s}|y(s)|,
\end{aligned}
$$

for all $v \in(-\infty, t], t \in(-\infty, \theta)$, which entails that

$$
e^{\lambda t}|y(t)| \leq \sup _{s \in(-\infty, t]} e^{\lambda s}|y(s)| \leq \frac{M\left(\|\phi\|_{\xi}+\varepsilon\right)}{1-P^{+} e^{\lambda \tau_{1}^{+}}} \quad \text { for all } t \in(-\infty, \theta) .
$$

Note that

$$
\begin{aligned}
Y^{\prime}(s)+Q(s) Y(s)= & -P(s) Q(s) y\left(s-\tau_{1}(s)\right) \\
& +\left[f\left(s, x\left(s-\tau_{2}(s)\right)\right)-f\left(s, x^{*}\left(s-\tau_{2}(s)\right)\right)\right], \\
s \in[0, t], t \in[0, \theta] . &
\end{aligned}
$$


Multiplying both sides of (3.17) by $e^{\int_{0}^{s} Q(u) d u}$, and integrating it on $[0, t]$, we get

$$
\begin{aligned}
Y(t)= & Y(0) e^{-\int_{0}^{t} Q(u) d u}+\int_{0}^{t} e^{-\int_{s}^{t} Q(u) d u}\left\{-P(s) Q(s) y\left(s-\tau_{1}(s)\right)\right. \\
& \left.+\left[f\left(s, x\left(s-\tau_{2}(s)\right)\right)-f\left(s, x^{*}\left(s-\tau_{2}(s)\right)\right)\right]\right\} d s, \quad t \in[0, \theta] .
\end{aligned}
$$

Thus, with the help of (3.8), (3.10), (3.11), (3.12), (3.14) and (3.16), we have

$$
\begin{aligned}
& |Y(\theta)|=\mid Y(0) e^{-\int_{0}^{\theta} Q(u) d u}+\int_{0}^{\theta} e^{-\int_{s}^{\theta} Q(u) d u}\left\{-P(s) Q(s) y\left(s-\tau_{1}(s)\right)\right. \\
& \left.+\left[f\left(s, x\left(s-\tau_{2}(s)\right)\right)-f\left(s, x^{*}\left(s-\tau_{2}(s)\right)\right)\right]\right\} d s \mid \\
& \leq|Y(0)| K^{*} e^{-\int_{0}^{\theta} \tilde{Q}(u) d u}+\int_{0}^{\theta} e^{-\int_{s}^{\theta} \tilde{Q}(u) d u} K^{*} \mid-P(s) Q(s) y\left(s-\tau_{1}(s)\right) \\
& +\left[f\left(s, x\left(s-\tau_{2}(s)\right)\right)-f\left(s, x^{*}\left(s-\tau_{2}(s)\right)\right)\right] \mid d s \\
& \leq\left(\|\phi\|_{\xi}+\varepsilon\right) K^{*} e^{-\int_{0}^{\theta} \tilde{Q}(u) d u}+\int_{0}^{\theta} e^{-\int_{s}^{\theta} \tilde{Q}(u) d u} K^{*}\left[|P(s) Q(s)|\left|y\left(s-\tau_{1}(s)\right)\right|\right. \\
& \left.+L^{f}\left|y\left(s-\tau_{2}(s)\right)\right|\right] d s \\
& \leq\left(\|\phi\|_{\xi}+\varepsilon\right) K^{*} e^{-\int_{0}^{\theta} \tilde{\mathrm{Q}}(u) d u} \\
& +\int_{0}^{\theta} e^{-\int_{s}^{\theta} \tilde{Q}(u) d u} K^{*}\left[\frac{M\left(\|\phi\|_{\xi}+\varepsilon\right) e^{-\lambda s}}{1-P^{+} e^{\lambda \tau_{1}^{+}}}|P(s) Q(s)| e^{\lambda \tau_{1}^{+}}\right. \\
& \left.+L^{f} \frac{M\left(\|\phi\|_{\xi}+\varepsilon\right)}{1-P^{+} e^{\lambda \tau_{1}^{+}}} e^{-\lambda s} e^{\lambda \tau_{2}^{+}}\right] d s \\
& =\left(\|\phi\|_{\xi}+\varepsilon\right) e^{-\lambda \theta} K^{*} e^{-\int_{0}^{\theta}[\tilde{Q}(u)-\lambda] d u} \\
& +\int_{0}^{\theta} e^{-\int_{s}^{\theta}[\tilde{Q}(u)-\lambda] d u} K^{*} \frac{1}{1-P^{+} e^{\lambda \tau_{1}^{+}}}\left[e^{\lambda \tau_{1}^{+}}|P(s) Q(s)|\right. \\
& \left.+L^{f} e^{\lambda \tau_{2}^{+}}\right] d s M\left(\|\phi\|_{\xi}+\varepsilon\right) e^{-\lambda \theta} \\
& \leq\left(\|\phi\|_{\xi}+\varepsilon\right) e^{-\lambda \theta} K^{*} e^{-\int_{0}^{\theta}[\tilde{Q}(u)-\lambda] d u} \\
& +\int_{0}^{\theta} e^{-\int_{s}^{\theta}[\tilde{Q}(s)-\lambda] d u}[\tilde{Q}(u)-\lambda] d s M\left(\|\phi\|_{\xi}+\varepsilon\right) e^{-\lambda \theta} \\
& =M\left(\|\phi\|_{\xi}+\varepsilon\right) e^{-\lambda \theta}\left[\left(\frac{K^{*}}{M}-1\right) e^{-\int_{0}^{\theta}(\tilde{Q}(u)-\lambda) d u}+1\right] \\
& <M\left(\|\phi\|_{\xi}+\varepsilon\right) e^{-\lambda \theta},
\end{aligned}
$$

which contradicts the fact that $|Y(\theta)|=M\left(\|\phi\|_{\xi}+\varepsilon\right) e^{-\lambda \theta}$. Hence, (3.13) holds. Letting $\varepsilon \longrightarrow$ $0^{+}$entails that

$$
\|Y(t)\| \leq M\|\phi\|_{\xi} e^{-\lambda t} \quad \text { for all } t>0
$$

Then, arguing as in the proof of (3.15) and (3.16), in view of (3.18), we can show

$$
e^{\lambda t}|y(t)| \leq \sup _{s \in(-\infty, t]} e^{\lambda s}|y(s)| \leq \frac{M\|\varphi\|_{\xi}}{1-P^{+} e^{\lambda \tau_{1}^{+}}},
$$


and

$$
|y(t)| \leq \frac{M\|\phi\|_{\xi}}{1-P^{+} e^{\lambda \tau_{1}^{+}}} e^{-\lambda t} \quad \text { for all } t>0,
$$

which completes the proof.

Remark 3.1 The results on periodic solutions or almost periodic solutions of (1.2) in references [1-11] are established under the condition that the decay term coefficient function $Q(t)$ is not oscillating. In this paper, the assumption $\left(A_{1}\right)$ relaxes the above technical condition. In fact, one can see Example 4.1 and Remark 4.1 for details.

\section{An example and its numerical simulations}

Example 4.1 Consider the following first-order neutral differential equations with timevarying delays and coefficients:

$$
\begin{aligned}
{[x(t)} & \left.-\frac{|\sin t|}{100} x(t-(4+\sin t+\sin \sqrt{2} t))\right]^{\prime} \\
= & -(1+2 \sin 200 t) x(t)+10 \cos \sqrt{2} t+10 \cos t+10 e^{-2 t^{4} \sin ^{2} t}+e^{\cos \sqrt{2} t} \\
& +\frac{1}{100} x\left(t-\left(1+\sin ^{2} \sqrt{3} t\right)\right),
\end{aligned}
$$

where

$$
\left\{\begin{array}{l}
P(t)=\frac{|\sin t|}{100}, \quad P^{+}=\frac{1}{100}, \quad \tau_{1}(t)=4+\sin t+\sin \sqrt{2} t, \quad \tau_{2}(t)=1+\sin ^{2} \sqrt{3} t \\
Q(t)=1+2 \sin 200 t, \quad Q^{+}=3, \quad \tilde{Q}(t)=1, \quad M[Q]=1, \quad K^{*}=e^{\frac{1}{50}}, \\
f(t, x)=10 \cos \sqrt{2} t+10 \cos t+10 e^{-2 t^{4} \sin ^{2} t}+e^{\cos \sqrt{2} t}+\frac{1}{100} x, \\
L^{f}=\frac{1}{100}, \quad L=\frac{1}{25} .
\end{array}\right.
$$

Then

$$
\sup _{t \in \mathbb{R}} K^{*} \frac{|Q(t) P(t)|+L^{f}}{\tilde{Q}(t)} \leq \frac{1}{25} e^{\frac{1}{50}}, \quad L+P^{+} \leq \frac{1}{20},
$$

and

$$
\sup _{t \in \mathbb{R}}\left\{-\tilde{Q}(t)+K^{*} \frac{1}{1-P^{+}}\left[|P(t) Q(t)|+L^{f}\right]\right\}<-0.6,
$$

which imply that (4.1) satisfies all conditions in Theorem 3.1. Hence, Eq. (4.1) has exactly one positive almost periodic solution $x^{*}(t)$. The corresponding simulation results of the solutions are seen in Fig. 1.

Remark 4.1 It should be mentioned that there is no research on the global exponential convergence of the pseudo-almost periodic solution for first-order neutral differential equations with time-varying delays and coefficients. Moreover, because $Q(t)=1+$ $2 \sin 200 t$ is oscillating on $\mathbb{R}$, one can see that all results in Refs. [1-11] cannot be applied to illustrate that all solutions for (4.1) converge exponentially to $x^{*}(t)$. We all know 


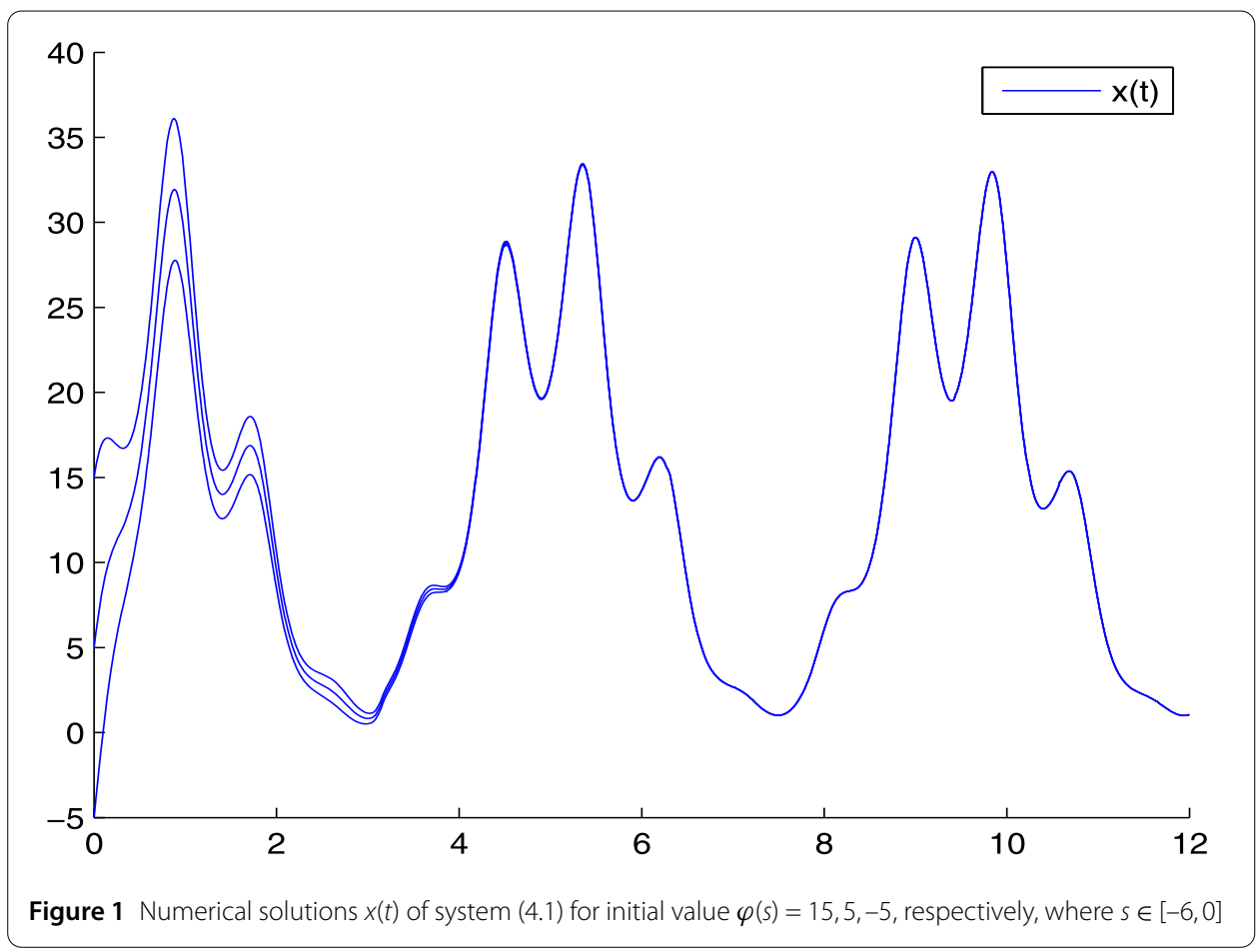

that the pseudo-almost periodic functions contain almost periodic functions, thus, the derived results are still novel if we reduce all time-varying delays and coefficients of (1.2) to periodic functions or almost periodic functions.

\section{Acknowledgements}

We would like to thank the anonymous referees for their valuable comments, which led to an improvement in the presentation. This work was supported by the Natural Scientific Research Fund of Hunan Provincial of China (Grant Nos. 2018JJ2087, 2018JJ2372), Natural Scientific Research Fund of Hunan Provincial Education Department of China (Grant No. 17C1076), Zhejiang Provincial Natural Science Foundation of China (Grant No. LY18A010019) and Zhejiang Provincial Education Department Natural Science Foundation of China (Y201533862).

\section{Competing interests}

The authors declare that they have no competing interests.

\section{Authors' contributions}

YHY and SHG worked together in the derivation of the mathematical results. Both authors read and approved the final manuscript.

\section{Author details}

${ }^{1}$ College of Mathematics and Computer Science, Hunan University of Arts and Science, Changde, P.R. China. ${ }^{2}$ College of Mathematics, Physics and Information Engineering, Jiaxing University, Jiaxing, P.R. China.

\section{Publisher's Note}

Springer Nature remains neutral with regard to jurisdictional claims in published maps and institutional affiliations.

\section{Received: 9 July 2017 Accepted: 20 March 2018 Published online: 27 March 2018}

\section{References}

1. Komanovskii, V.B., Nosov, V.R.: Stability of Functional Differential Equations. Academic Press, London (1986)

2. Kuang, Y.: Delay Differential Equations with Applications in Population Dynamical System. Academic Press, New York (1993)

3. Liu, B., Huang, L.: Existence and uniqueness of periodic solutions for a kind of first order neutral functional differential equations. J. Math. Anal. Appl. 322(1), 121-132 (2006)

4. Liu, B., Huang, L.: Existence and uniqueness of periodic solutions for a kind of first order neutral functional differential equations with a deviating argument. Taiwan. J. Math. 11(2), 497-510 (2007) 
5. Luo, Y., Wang, W., Shen, J.: Existence of positive periodic solutions for two kinds of neutral functional differential equations. Appl. Math. Lett. 21, 581-587 (2008)

6. Graef, J.R., Kong, L.: Periodic solutions of first order functional differential equations. Appl. Math. Lett. 24, 1981-1985 (2011)

7. Olach, R.: Positive periodic solutions of delay differential equations. Appl. Math. Lett. 26, 1141-1145 (2013)

8. Peng, L., Wang, L.: Periodic solutions for first order neutral functional differential equations with multiple deviating arguments. Ann. Pol. Math. 111(2), 197-213 (2014)

9. Candan, T:: Existence of positive periodic solutions of first order neutral differential equations with variable coefficients. Appl. Math. Lett. 52, 142-148 (2016)

10. Diagana, T., Hernández, E.M.: Existence and uniqueness of pseudo almost periodic solutions to some abstract partial neutral functional differential equations and applications. J. Math. Anal. Appl. 327(2), 776-791 (2007)

11. Diagana, T., Hernández, E.M., José dos Santos, P.C.: Existence of asymptotically almost automorphic solutions to some abstract partial neutral integro-differential equations. Nonlinear Anal. 71(1-2), 248-257 (2009)

12. Liu, B.: Pseudo almost periodic solutions for neutral type CNNs with continuously distributed leakage delays. Neurocomputing $148,445-454$ (2015)

13. Liu, B.: Pseudo almost periodic solutions for CNNs with continuously distributed leakage delays. Neural Process. Lett. 42, 233-256 (2015)

14. Shao, J.: Pseudo almost periodic solutions for a Lasota-Wazewska model with an oscillating death rate. Appl. Math. Lett. 43, 90-95 (2015)

15. Yu, Y: Exponential stability of pseudo almost periodic solutions for cellular neural networks with multi-proportional delays. Neural Process. Lett. 45, 141-151 (2017)

16. Xiong, W: New results on positive pseudo-almost periodic solutions for a delayed Nicholson's blowflies model. Nonlinear Dyn. 85, 563-571 (2016)

17. Huang, Z:: Almost periodic solutions for fuzzy cellular neural networks with time-varying delays. Neural Comput. Appl. 28, 2313-2320 (2017)

18. Huang, Z: Almost periodic solutions for fuzzy cellular neural networks with multi-proportional delays. Int. J. Mach. Learn. Cybern. 8, 1323-1331 (2017)

19. $\mathrm{Xu}, \mathrm{Y}$ :: Exponential stability of pseudo almost periodic solutions for neutral type cellular neural networks with $D$ operator. Neural Process. Lett. 46, 329-342 (2017)

20. Zhang, H.: New results on the positive pseudo almost periodic solutions for a generalized model of hematopoiesis. Electron. J. Qual. Theory Differ. Equ. 2014(24), 1 (2014)

21. Fink, A.M.: Almost Periodic Differential Equations. Lecture Notes in Mathematics, vol. 377. Springer, Berlin (1974)

22. Zhang, C.: Almost Periodic Type Functions and Ergodicity. Kluwer Academic, Beijing (2003)

23. Zhang, C.: Pseudo almost periodic solutions of some differential equations II. J. Math. Anal. Appl. 192, 543-561 (1995)

24. Hale, J.K.: Ordinary Differential Equations. Krieger, Malabar (1980)

\section{Submit your manuscript to a SpringerOpen ${ }^{\circ}$ journal and benefit from:}

- Convenient online submission

- Rigorous peer review

- Open access: articles freely available online

- High visibility within the field

- Retaining the copyright to your article

Submit your next manuscript at $\boldsymbol{\nabla}$ springeropen.com 